\author{
UNIVERSIDADE DE SÃO PAULO \\ HOSPITAL DE REABILITAÇÃO DE ANOMALIAS CRANIOFACIAS
}

ANDREIA FERNANDES EMILIO LAPOSTA

Avaliação da simetria dos arcos dentários em pacientes com fissura labiopalatina reabilitados com prótese parcial fixa na região da fenda, após tratamento ortodôntico 



\title{
Avaliação da simetria dos arcos dentários em pacientes com fissura labiopalatina que reabilitados com prótese parcial fixa na região da fenda, após tratamento ortodôntico
}

\begin{abstract}
Dissertação constituída por artigo apresentada ao Hospital de Reabilitação de Anomalias Craniofaciais da Universidade de São para obtenção do título de Mestre em Ciências da Reabilitação, na área de concentração Fissuras Orofaciais e Anomalias Relacionadas.
\end{abstract}

Orientadora: Profa. Dra. Simone Soares 
Laposta, Andreia Fernandes Emilio

Avaliação da simetria dos arcos dentários em pacientes com fissura labiopalatina reabilitados com prótese parcial fixa na região da fenda, após tratamento ortodôntico / Andréia Fernandes Emílio Laposta. -Bauru, 2021.

59 p. : il. ; $31 \mathrm{~cm}$.

Dissertação (mestrado) -- Hospital de Reabilitação de Anomalias Craniofaciais, Universidade de São Paulo, ano de defesa.

Orientadora: Profa. Dra. Simone Soares

Autorizo, exclusivamente para fins acadêmicos e científicos, a reprodução total ou parcial desta dissertação/tese, por processos fotocopiadores e outros meios eletrônicos.

Afromizis

Andreia Fernandes Emilio Laposta

Data: 22/02/2021

Comitê de Ética do HRAC-USP

Protocolo n: 4.263 .528

Data: 08/09/2020 
ERRATA 

FOLHA DE APROVAÇÃO 



\section{DEDICATÓRIA}

Dedico este trabalho, com muito carinho, aos meus pais João e Alice, por me incentivarem e me dar força neste momento, e toda minha família minha eterna gratidão.

A todos que me acompanharam durante o período de realização deste trabalho.

Em especial minha querida Amiga Adriana, minha maior incentivadora para fazer Mestrado, conseguimos vencer mais essa batalha sem medir esforços, obrigada por tanto. 



\section{AGRADECIMENTOS}

Agradeço a Deus, pela força e coragem durante toda esta longa caminhada.

Um eterno sentimento de gratidão a minha querida Orientadora Dra. Simone Soares, pela oportunidade de realizar este trabalho, ao lado de alguém que transpira sabedoria, meu respeito e admiração pelo seu ensinamento desde a graduação.

À Secretária do Programa de Pós Graduação do HRAC-USP, pelo apoio e paciência em todos os momentos.

Aos meus amigos e amigas de curso e trabalho que fizeram parte da minha formação e que vão continuar presentes em minha vida e em meu coração. 

"Quando penso que cheguei ao meu limite descubro que tenho forças para ir além".

Ayrton Senna 

O presente trabalho avaliou a simetria dos arcos dentários de pacientes com e sem fissura labiopalatina através da comparação e correlação entre os lados direito e esquerdo dos arcos dentários (maxila e mandíbula), após tratamento ortodôntico/reabilitador utilizando uma prótese parcial fixa de 3 elementos na região da fenda. O estudo transversal, observacional, retrospectivo incluiu 15 pacientes com fissura labiopalatina submetidos a tratamento ortodôntico e reabilitados com uma prótese parcial fixa (GPPF) e 20 pacientes sem fissura labiopalatina submetidos a tratamento ortodôntico (GNF). Todos os pacientes $(n=35)$ foram inicialmente diagnosticados com mordida cruzada anterior e/ou posterior e submetidos a mecânica ortodôntica semelhante. Os critérios de exclusão foram cirurgia ortognática, presença de síndromes e documentação incompleta. A simetria do arco foi avaliada utilizandose modelos dentários digitalizados com um scanner a laser (R700TM; 3Shape). Três medidas lineares foram determinadas em cada um dos lados (direito e esquerdo): distância incisivo-canino (INC), canino-molar (CM) e incisivo-molar (INM) avaliadas através do programa Vectra Analysis Module (VECTRA H1; Canfield Scientific, Inc). O teste t-pareado e o coeficiente de correlação de Pearson ( $r$-value), significância $\alpha=$ 0.05, foram utilizados para comparação entre os lados direito e esquerdo do arco, de acordo com o fator grupo e sexo. No grupo GPPF o teste t-pareado não apresentou nenhuma diferença em nenhum dos lados em nenhum arco, entretanto a única região que apresentou correlação moderada foi a região posterior de mandíbula $(\mathrm{CM}: r=0,56$; INM, r=0,57). Já no grupo GNF, tanto a maxila quanto a mandíbula foram consideradas simétricas apresentando correlação forte em todas as medidas lineares. No grupo GPPF, o sexo feminino apresentou correlação moderada na maxila em duas das três medidas lineares avaliadas (INC e CM: $r=0,57$ e $r=0,59$, respectivamente) e forte em todas as medidas lineares mandibulares (INC, CM e INM: $r=0,82, r=0,69$ e $r=0,78$, respectivamente). Já no sexo masculino nenhuma medida linear apresentou correlação. O grupo GNF tanto no sexo feminino como no masculino, a maxila foi considerada simétrica e somente na mandíbula do sexo masculino na região posterior não se verificou correlação (CM e INM: $r=0,38$ e $r=0,55$, respectivamente). Os pacientes que receberam uma prótese fixa, na região anterior de maxila, não 

apresentaram simetria desejada após o tratamento, sendo que esse resultado foi obtido devido a assimetria verificada no sexo masculino.

Palavras-chave: Fenda labial. Fissura Palatina. Estética. Reabilitação. Reabilitação Bucal. 



\section{ABSTRACT \\ Symmetry evaluation of dental arch in cleft lip and palate patients rehabilitated with a fixed partial denture in the cleft region after orthodontic treatment}

The present study aimed to evaluate the dental arch symmetry in cleft and non-cleft patients by comparing and correlating the right and left sides of dental arches (maxilla and mandible) after orthodontic/rehabilitative treatment with a three-unit fixed partial dental prosthesis in the cleft region. The cross-sectional, observational, retrospective study included 15 patients with cleft lip and palate undergoing orthodontic treatment and rehabilitated with a fixed partial denture (GPPF) and 20 patients without cleft lip and palate undergoing orthodontic treatment (GNF). All patients $(n=35)$ were initially diagnosed with anterior and/or posterior crossbite and underwent similar orthodontic mechanics. Exclusion criteria were orthognathic surgery, presence of syndromes also incomplete documentation. Exclusion criteria were orthognathic surgery, presence of syndromes and incomplete documentation. The arch symmetry was assessed using dental models digitized with a laser scanner (R700TM; 3Shape). Three linear measurements were established on each side (right and left): incisive-canine (INC), canine-molar (CM), and incisive-molar (INM) distance assessed using the Vectra Analysis Module program (VECTRA H1; Canfield Scientific, Inc). According to group and sex factor, the paired t-test and Pearson's correlation coefficient ( $r$-value) compared the right and left sides of the arch with a significance level of $5 \%$. In the GPPF group, the paired t-test did not show any difference on either side in any arch, however, the only region that showed a moderate correlation was the posterior mandible region ( $\mathrm{CM}: r=0.56$; INM, $r=0.57)$. In the GNF group, both the maxilla and the mandible were considered symmetrical, showing a strong correlation in all linear measurements. The female gender in the GPPF group showed a moderate correlation in the maxilla in two of the three linear measures evaluated (INC and CM: $r=0.57$ and $r=0.59$, respectively) and strong in all the mandibular linear measurements (INC, CM, and INM: $r=0.82, r=0.69$ and $r=0.78$, respectively). In the male sex, no linear measure showed a correlation. In the GNF group in both males and females, the maxilla was considered symmetrical, and only in the male mandible in the posterior region, there was no correlation (CM and 

INM: $r=0.38$ and $r=0.55$, respectively). Patients who received a fixed prosthesis, in the maxilla anterior area did not present desired symmetry after treatment, and this result was obtained by the asymmetry observed in males.

Keywords: Cleft lip. Cleft palate. Aesthetics. Rehabilitation. Oral Rehabilitation. 



\section{LISTA DE ILUSTRAÇÕES}

Figura 1 - Medidas Lineares ............................................................ 36 



\section{LISTA DE TABELAS}

Tabela 1 - Comparação e correlação das medidas lineares entre os lados direito e esquerdo, em pacientes com fissura labiopalatina (GPPF) e sem fissura (GNF).

Tabela 2 - Comparação e correlação das medidas lineares entre os lados direito e esquerdo em pacientes com fissura labiopalatina (GPPF) de acordo com o sexo................................................. 38

Tabela 3 - Comparação e correlação das medidas lineares entre os lados direito e esquerdo em pacientes sem fissura (GNF) de acordo com o sexo 

SUMÁRIO

\begin{tabular}{|c|c|}
\hline 1 & INTRODUÇÃO \\
\hline 2 & OBJETIVOS ................ \\
\hline 2.1 & OBJETIVO GERAL \\
\hline 2.2 & OBJETIVOS ESPECÍFICOS \\
\hline 3 & ARTIGOON) \\
\hline 4 & CONCLUSÃO GERAL. \\
\hline & REFERÊNCIAS...... \\
\hline
\end{tabular}





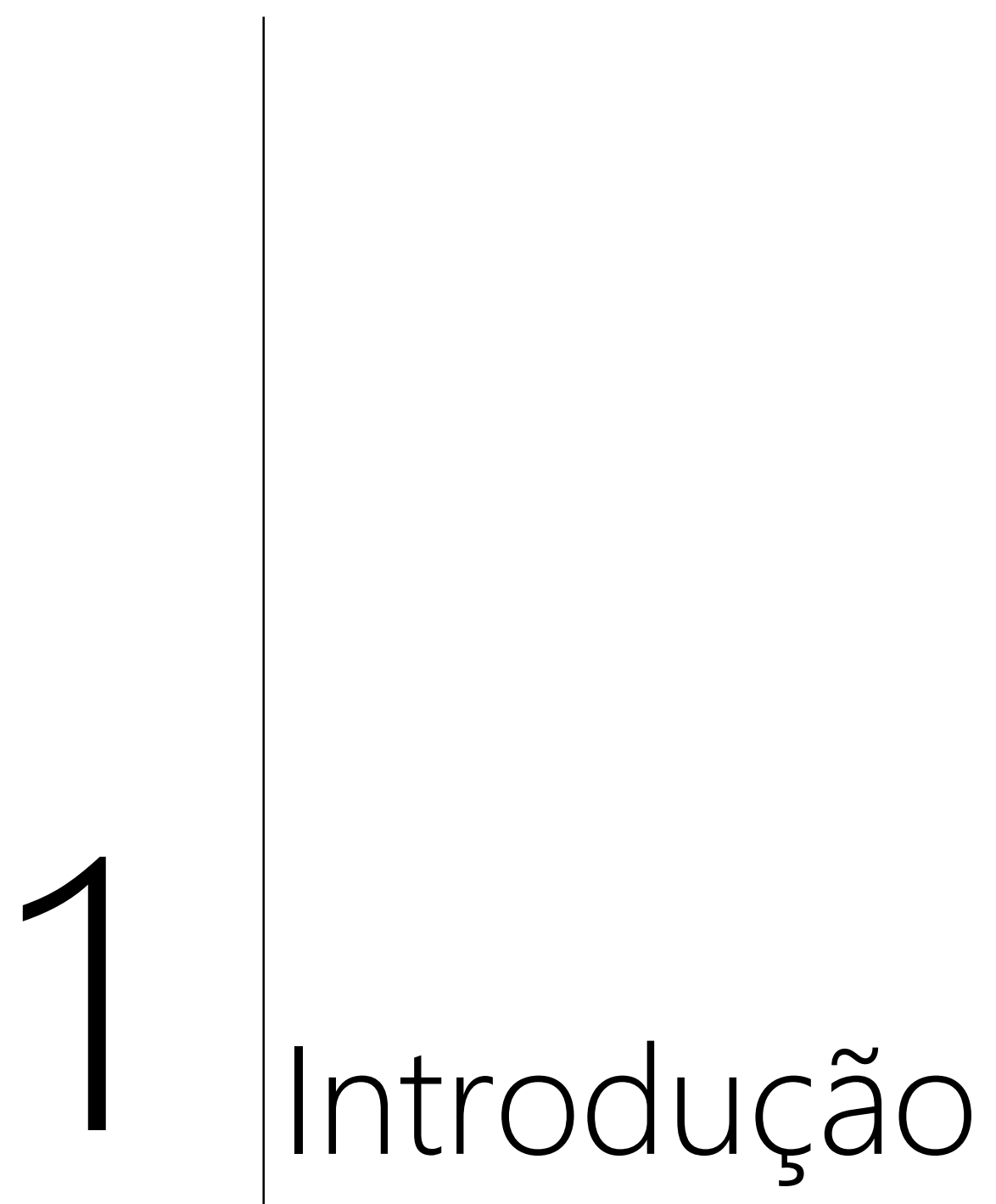





\section{INTRODUÇÃO}

O desenvolvimento facial embrionário humano é complexo (SHKOUKANI et al., 2014) e as anomalias craniofaciais podem variar desde pequenas assimetrias até grandes defeitos faciais com consequências anatômicas, funcionais e estéticas, que, na dependência de sua gravidade, comprometerão em menor ou maior proporção, o desenvolvimento do complexo bucomaxilofacial (WILL, 2000).

Uma das anomalias craniofaciais mais comuns são as fissuras labiopalatinas, consideradas alterações anatômicas congênitas que resultam na não fusão dos processos embrionários e palatinos, que deveria ocorrer entre a $4^{\underline{a}}$ e $12^{a}$ semana de vida intrauterina, quando acontece a formação dos arcos faríngeos (KATCHBURIAN; ARANA, 2012). O desenvolvimento natural do lábio e palato envolve uma série de acasos intimamente planejados durante o desenvolvimento dos primórdios faciais que incluem migração celular, crescimento, diferenciação e apoptose. Transtornos nesses eventos, por meio de fatores genéticos e ambientais, podem afetar a morfogênese adequada das estruturas faciais (BUTALI et al., 2011).

As fissuras labiopalatinas apresentam prevalência estimada de aproximadamente $1 \mathrm{em} 700$ nascidos vivos, com variações étnicas e geográficas, sendo considerada um relevante problema de saúde pública, segundo a Organização Mundial de Saúde (WORLD HEALTH ORGANIZATION, 2002).

As deformidades das fendas orofaciais podem ser sindrômicas ou não sindrômicas, por natureza. As fissuras sindrômicas são aquelas associadas as malformações envolvendo outras regiões de desenvolvimento, já as não sindrômicas podem ser anomalias isoladas ou podem estar associadas a outras anomalias de desenvolvimento ou malformação primária.

Pela gravidade variável, complexidade e diversidade morfológica das fissuras orofaciais, diferentes classificações foram propostas com a finalidade de padronizar seu diagnóstico e o protocolo de tratamento (TOLAROVÁ; CERVENKA, 1998).

No Hospital de Reabilitação de Anomalias Craniofaciais, Universidade de São Paulo (HRAC/USP), adota-se a classificação de Spina et al. (1972) modificada por Silva Filho, Ramos e Abdo (1992). Essa classificação se baseia na evolução embriológica dos tecidos envolvidos na formação orofacial e na morfologia da face, tendo o forame incisivo como referência anatômica, já que simboliza o único vestígio 
do que separava, na vida intrauterina, o palato primário do palato secundário (SILVA FILHO; FREITAS, 2007). Desta forma, as fissuras labiopalatinas se dividem em fissuras pré-forame incisivo, fissuras transforame incisivo, fissura pós-forame incisivo e fissuras raras da face. Ainda há subdivisões, podendo ser fissuras unilaterais, bilaterais ou medianas, completas ou incompletas. As fissuras podem estar associadas a uma grande quantidade de síndromes, constituindo de 5 a $7 \%$ do total de fissuras (FREITAS et al., 2012a).

As fissuras que envolvem o palato primário acarretam implicações estéticas permanentes na face e, quando passam pelo rebordo alveolar, interferem na oclusão. Já as que acometem o palato secundário desencadeiam problemas funcionais relacionados a fala e audição.

O momento apropriado e a ordem dos cuidados específicos relacionados a fissura são fatores importantes para otimizar os resultados, no entanto, o cuidado deve ser individualizado para atender as necessidades específicas de cada paciente e família (LEWIS; JACOB; LEHMANN, 2017).

Os diversos envolvimentos estéticos e funcionais devido à presença da fissura labiopalatina, requerem reabilitação (FREITAS et al., 2012a) e os indivíduos que apresentam essa alteração necessitam de acompanhamento multidisciplinar ao longo da vida, desde o nascimento até a idade adulta (MARTELLI et al., 2012). Não se pode definir um tempo exato para a reabilitação completa, pois cada paciente apresenta um tipo de fissura e uma situação que requer intervenções diferenciadas (ALONSO et al., 2009).

Assim, o processo de reabilitação desses pacientes é necessário para que o indivíduo seja incluído na sociedade e tenha sua dignidade restaurada, porém é longo e complexo. Constituído por inúmeras etapas e abrangendo as mais variadas especialidades (BARDACH et al., 1985) tendo idealmente seu início logo nos primeiros dias após o nascimento, com as cirurgias plásticas primárias de queiloplastia e palatoplastia (BERTIER; TRINDADE; SILVA FILHO, 2007; FREITAS et al., 2012b; 2012c). As cirurgias primárias são realizadas em tecido mole, com o objetivo de reconstruir os defeitos presentes no lábio e no palato respectivamente (BERTIER; TRINDADE; SILVA FILHO, 2007; SILVA FILHO; FREITAS et al., 2012b; 2012c).

Conforme o protocolo do Hospital de Reabilitação de Anomalias Craniofaciais (HRAC/USP), a cirurgia reparadora do lábio (queiloplastia), é realizada por volta dos 3 meses de vida, e a cirurgia reparadora do palato, (palatoplastia), é 
realizada em torno dos 12 meses. Essas cirurgias, apesar de promoverem uma melhora estética e funcional do paciente, interferem na qualidade do crescimento craniofacial. Principalmente nos indivíduos com fissuras com envolvimento total de lábio, rebordo alveolar e palato (SEMB; SHAW, 1991; SEMB et al., 2005; GNOINSKI; RUTZ, 2009; KREY; BÖRNGEN; DANNHAUER, 2009).

A fissura do processo alveolar maxilar é reparada entres os 9 e 12 anos de idade, de acordo com o desenvolvimento da dentição. Enxertos ósseos autógenos são colocados para unir os segmentos da maxila, e todas as fístulas orofaciais são fechadas. Os procedimentos seguintes podem incluir cirurgia ortognática e revisão labial ou nasal (ABRAMSON et al., 2015).

A odontologia (ortodontia), assim como a cirurgia plástica e a fonoaudiologia, constituem os pilares essenciais na reabilitação da fissura labiopalatina. A ortodontia e a cirurgia bucomaxilofacial, entre as diversas especialidades odontológicas, representam parte do tratamento desejado pelos pacientes, pois corrigem deficiências que comprometem a estética facial, incluindo a má oclusão dentária e alterações esqueléticas faciais (FREITAS et al., 2012a)

Todo período de crescimento, do nascimento até à idade adulta de indivíduos com fissura labiopalatina, requer um protocolo de documentação clínica sistemática, para um planejamento apropriado e prospectivo por meio do ajuste do manejo nas várias fases do tratamento, e da avaliação longitudinal do progresso do tratamento (WUTZL et al., 2009; MELLO et al., 2013). Frequentemente, essas avaliações do crescimento do complexo maxilo-mandibular são realizadas por análise em modelos de gesso (ESTEVES; BOMMARITO, 2007) ou por exames de imagem, tais como fotografias, radiografias cefalométricas (SABARINATH et al., 2010; ROUSSEAU et al., 2013) e tomografias (KIM et al., 2012; OTERO et al., 2012; YANG et al., 2012). Apesar de efetivos, pelo posicionamento do paciente, mas também pela distorção de imagem e sobreposição de estruturas, podem levar a erros, dificultando a análise mais objetiva das informações (MENEZES et al., 2016). Entretanto, a inadequação em relação aos modelos de estudo em gesso, a estocagem, a manutenção, o acesso e a troca de informações inter centros (ROSATI et al., 2012) levaram ao desenvolvimento de métodos tecnológicos acessíveis para facilitar a avaliação morfológica das estruturas em crescimento, bem como preencher as necessidades atuais. Assim a digitalização de modelos e análise tridimensional das arcadas dentárias representam uma mudança expressiva na documentação dos 
dados anatômicos e estruturais desses pacientes (SFORZA et al., 2012; HUANCA GHISLANZONI et al., 2013; MELLO et al., 2013; KUIJPERS et al., 2014; UGOLINI et al., 2015; MENEZES et al., 2016)

O tratamento reabilitador é desafiador, pois as alterações anatômicas e funcionais estão diretamente relacionadas ao tipo de malformações e à idade do início do tratamento. Por isso o tratamento de escolha para reabilitar a área com fissura é a ortodontia. A obtenção da oclusão ideal e relações intermaxilares, assim como a manutenção dos resultados obtidos, como alinhamento apropriado dos dentes em ambas as arcadas dentárias, com sobressaliência e sobremordida adequadas, são desejados porque proporcionam custos reduzidos e manutenção mais simples ao longo da vida do paciente. Porém, o espaço edêntulo remanescente nem sempre é fechado pelo tratamento ortodôntico. Quando o osso alveolar está ausente, a movimentação ortodôntica não é possível ou não pode fornecer resultados funcionais e estéticos suficientes para o tratamento. Nesses casos, a reabilitação dos incisivos laterais é necessária após a manutenção ortodôntica do espaço edêntulo. As alternativas de reabilitação com próteses variam, proporcionando maior satisfação psicológica, e devem ser consideradas (MALGAJ et al., 2020). Dessa forma, muitos pacientes necessitam de diferentes tipos de próteses dentárias para reabilitar a área da fenda edêntula, normalmente a área dos incisivos laterais (CABALLERO et al., 2019; PUCCIARELLI et al., 2020). A intervenção reabilitadora com próteses fixas se tornou uma necessidade quando a extensão edêntula permanece após a conclusão do tratamento ortodôntico e geralmente é considerada um elo final para a reabilitação oral completa em paciente com fissura labiopalatina (FREITAS et al., 2013). A possibilidade de tratamento escolhida para restaurar o local edêntulo em paciente com fissura labiopalatina é prescrita pela patência e extensão do defeito, presença de fenda labial e ou palatina não reparada, relação maxilomandibular e estado dos dentes remanescentes (BHANDARI, 2017).

As próteses parciais fixas convencionais são bem indicadas quando há limitação do tamanho da fissura para uso de implantes ou quando o enxerto ósseo foi perdido (PINTO; LOPES, 2007). As próteses podem promover contorno facial mais adequado e quando bem planejadas, levam a estética e a função satisfatória, permitindo que os pacientes fiquem mais positivos com a autoimagem após a reabilitação (AYNA; BAŞARAN; BEYDEMIR, 2009). 
Independentemente do tipo de prótese a ser usada na reabilitação, esta deve apresentar simetria com o dente adjacente, bem como mimetizar o dente natural para que se obter estética satisfatória (KAZOR et al., 2004; FÜRHAUSER et al., 2005; JUODZBALYS; WANG, 2007).

A etiologia da assimetria inclui: malformações genéticas ou congênitas, fatores ambientais (hábitos e traumas) e desvios funcionais. As assimetrias dentárias e desvios funcionais podem ser tratados ortodonticamente, o que não ocorre com as assimetrias faciais (BISHARA; BURKEY; KHAROUF, 1994). Durante a dentição mista, fatores ambientais podem explicar melhor a assimetria, porque as mudanças no crescimento e desenvolvimento são aceleradas após o período relativamente estável da dentição decídua (MAURICE; KULA, 1998; ŠLAJ et al., 2003). Em pacientes com fissura labiopalatina (MOLSTED; DAHL, 1990; PRECIOUS; DELAIRE, 1993; PARK; PARK; BAEK, 2018) as investigações da simetria do arco dentário após a maturidade esquelética são restritas. Porém, a simetria do arco dentário é uma referência importante para o tratamento ortodôntico de pacientes com fissura labiopalatina (SCHAEFER et al., 2006; CHEN et al., 2015), além de permitir a verificação dos resultados obtidos. Alguns estudos concluíram que a atividade muscular influencia e determina a forma dos arcos dentários, resultando em assimetria (LEAR, 1968). As regiões dento alveolares e mandibulares, segundo Shah e Joshi (1978) se mostraram simétricas, devido ao equilíbrio muscular da língua e forças labiais, o que pode não ser verificado nos pacientes com fissura labiopalatina, uma vez que há quebra desse equilíbrio, devido a presença da fissura. Contudo, Scanavini et al. (2012), observaram assimetrias em todos os arcos dentários dos indivíduos estudados, independente da presença de má-oclusão (SCANAVINI et al., 2012)

Diante do exposto, estudar e avaliar as simetrias pós tratamento ortodôntico e reabilitador protético é importante pois permite verificar a direção do tratamento proposto e ainda estabelecer objetivamente a presença ou não de possíveis alterações nos arcos dentários e trazer à luz da ciência novas perspectivas. 




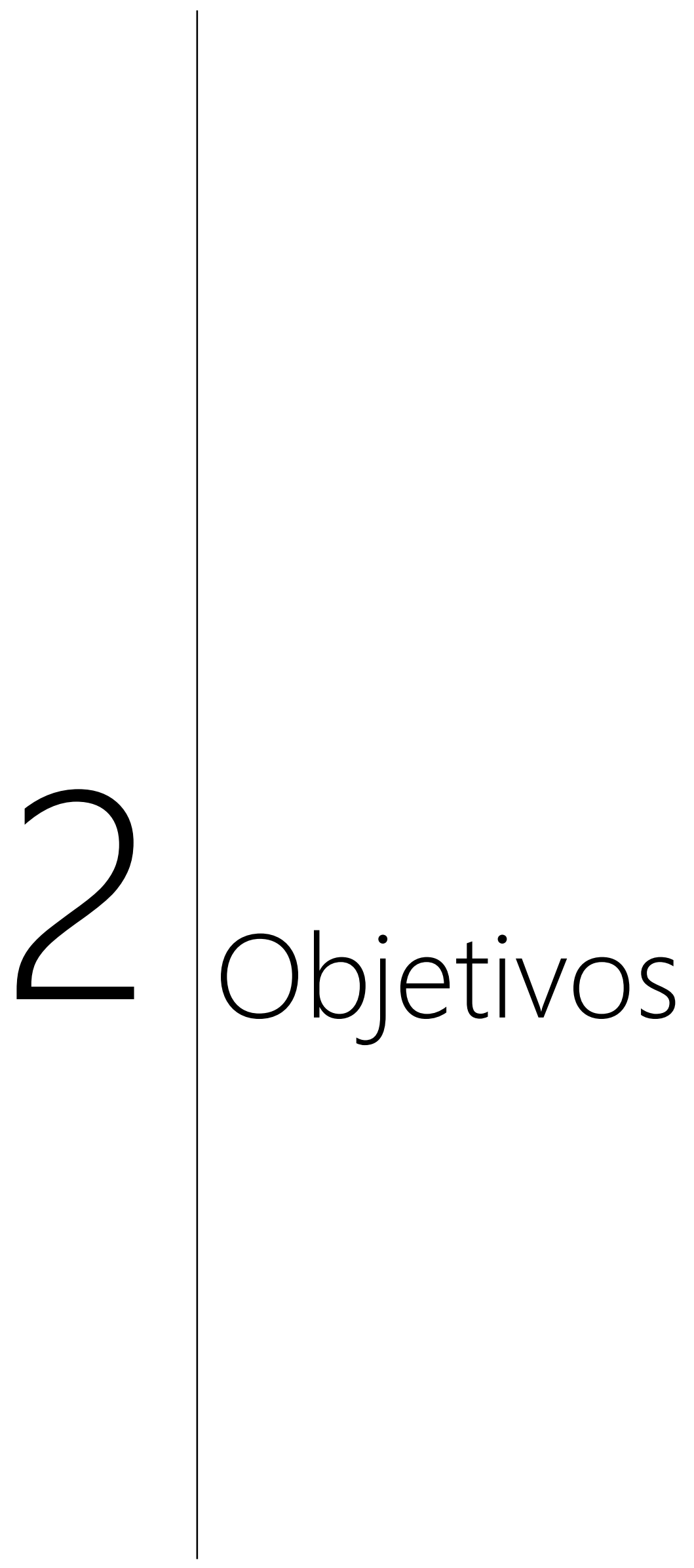






\section{OBJETIVOS}

\subsection{OBJETIVO GERAL}

O objetivo geral do trabalho foi avaliar a simetria dos arcos dentários (maxila e mandíbula) de pacientes com e sem fissura labiopalatina, através da comparação e correlação entre os lados direito e esquerdo dos arcos, após tratamento ortodôntico e protético, em pacientes que receberam uma prótese parcial fixa de 3 elementos na região da fenda.

\subsection{OBJETIVOS ESPECÍFICOS}

Analisar e comparar a simetria dos arcos dentários em duas variáveis, sendo a primeira variável o fator grupamento (pacientes com fissura labiopalatina e sem fissuras orofaciais) e a segunda variável de análise sendo o fator sexo, verificado nos dois grupos estudados. 



$$
3 \text { Artigo }
$$





\section{ARTIGO}

O artigo apresentado na dissertação foi escrito de acordo com as instruções e diretrizes para submissão de artigo da revista Journal of Craniofacial Surgery.

\section{Avaliação da simetria dos arcos dentários em pacientes com fissura labiopalatina reabilitados com prótese parcial fixa na região da fenda, após tratamento ortodôntico}

Andreia Fernandes Emilio Laposta ${ }^{1}$, José Antônio de Siqueira Laurenti ${ }^{1}$, Maria Giulia Rezende Pucciarelli ${ }^{2}$, Jefferson Freire Cardoso ${ }^{2}$, Karin Hermana Neppelenbroek ${ }^{3}$ Simone $^{2}$ Soares $^{4}$

${ }^{1} \mathrm{MSc}$, Postgraduate student, Hospital for Rehabilitation of Craniofacial Anomalies, University of São Paulo. Rua Sílvio Marchione 3-20 - Bauru - SP - Brasil - 17012-900.

${ }^{2} \mathrm{MSc}$, Postgraduate student, Bauru School of Dentistry, University of São Paulo. Al. Octávio Pinheiro Brisola, 9-75 - Bauru - SP - Brasil - 17012-901.

${ }^{3}$ Associate Professor, Department of Prosthodontics and Periodontology, Bauru School of Dentistry/USP. Al. Octávio Pinheiro Brisola, 9-75 - Bauru - SP - Brasil - 17012-901.

${ }^{4}$ Associate Professor, Department of Prosthodontics and Periodontology, Bauru School of Dentistry/USP, Hospital for Rehabilitation of Craniofacial Anomalies/USP. Al. Octávio Pinheiro Brisola, 9-75 - Bauru - SP - Brasil - 17012-901.

\section{${ }^{\star}$ Corresponding author:}

Simone Soares

Bauru School of Dentistry - University of São Paulo

Alameda Dr. Octávio Pinheiro Brisolla, 9-75

Bauru, São Paulo, 17012-901- Brazil

Phone: 551432358312 Fax: (14) 32234679

E-mail: sisoares@usp.br

\section{Fonte de Financiamento}

Este estudo foi financiado pela Fundação de Amparo a Pesquisa do Estado de São Paulo Bolsa FAPESP nำ 2016 / 14942-6

\section{Conflitos de interesse}


Os autores declararam explicitamente que não há conflitos de interesse em conexão com este artigo. 


\section{RESUMO}

O trabalho avaliou a simetria dos arcos dentários de pacientes com e sem fissura labiopalatina, após tratamento ortodôntico/reabilitador utilizando uma prótese parcial fixa de 3 elementos na região da fenda. Estudo transversal, observacional, retrospectivo incluiu 35 pacientes, 15 com fissura labiopalatina, submetidos a tratamento ortodôntico e reabilitados com uma prótese parcial fixa (GPPF) e 20 pacientes sem fissura labiopalatina, submetidos a tratamento ortodôntico (GNF). A simetria do arco foi avaliada utilizando-se modelos dentários digitalizados, medidas lineares foram determinadas nos lados direito e esquerdo: distância incisivo-canino (INC), canino-molar (CM) e incisivo-molar (INM). O teste t-pareado e o coeficiente de correlação de Pearson ( $r$-value), significância $\alpha=0.05$, foram utilizados para comparação entre os lados, de acordo com o fator grupo e sexo. No GPPF, o teste tpareado, a única região que apresentou correlação moderada foi a região posterior de mandíbula (CM: $r=0,56$; INM, $r=0,57)$. No $G N F$, os arcos foram considerados simétricos, correlação forte em todas as medidas. Quanto ao sexo, no GPPF, o feminino apresentou correlação moderada na maxila em duas das três medidas avaliadas (INC: $r=0,57$ e CM: $r=0,59$ ) e forte em todas as medidas mandibulares (INC: $r=0,82, C M: r=0,69$ e INM: $r=0,78)$. O GNF nos sexos, a maxila foi considerada simétrica e somente na mandíbula do sexo masculino na região posterior não se verificou correlação (CM: $r=0,38$ e INM: $r=0,55$ ). Os pacientes que receberam uma prótese fixa, na região anterior de maxila, não apresentaram simetria após 0 tratamento, sendo que esse resultado foi obtido devido a assimetria verificada no sexo masculino.

Palavras-chave: Fenda labial. Fissura Palatina. Estética. Reabilitação. Reabilitação Bucal 


\section{INTRODUÇÃO}

O desenvolvimento facial embrionário humano é complexo ${ }^{1} \mathrm{e}$ as anomalias craniofaciais podem variar desde pequenas assimetrias até grandes defeitos faciais com consequências anatômicas, funcionais e estéticas, que, na dependência de sua gravidade, comprometerão em menor ou maior proporção, o desenvolvimento do complexo bucomaxilofacial. ${ }^{2}$ As fissuras labiopalatinas são alterações anatômicas congênitas, com prevalência de 1 em 700 nascido vivos e relevante problema de saúde pública. ${ }^{3}$

Os diversos envolvimentos estéticos e funcionais devido à presença da fissura labiopalatina, requerem reabilitação ${ }^{4}$ e acompanhamento multidisciplinar ao longo da vida, desde o nascimento até a idade adulta. ${ }^{5}$ Assim, o processo de reabilitação desses pacientes é necessário para que o indivíduo seja incluído na sociedade e tenha sua dignidade restaurada, porém é longo e complexo.

A odontologia (ortodontia), assim como a cirurgia plástica e a fonoaudiologia, constituem os pilares essenciais na reabilitação da fissura labiopalatina. ${ }^{6}$

Todo período de crescimento, dos indivíduos com fissura labiopalatina, requer um protocolo de documentação clínica sistemática, para um planejamento apropriado e prospectivo por meio do ajuste do manejo nas várias fases do tratamento, e da avaliação longitudinal do progresso do tratamento. ${ }^{7-8} \mathrm{Com}$ a evolução tecnológica novos sistemas se tornaram acessíveis para facilitar a avaliação morfológica das estruturas em crescimento, bem como preencher as necessidades atuais. Assim a digitalização de modelos e análise tridimensional das arcadas dentárias representam uma mudança expressiva na documentação dos dados anatômicos e estruturais desses pacientes. ${ }^{8-13}$

O tratamento ideal para reabilitar os pacientes com fissura labiopalatina é a ortodontia, mas nem sempre é possível se atingir o objetivo proposto e o espaço edêntulo nem sempre é reabilitado. Quando o osso alveolar está ausente, a movimentação ortodôntica não é possível ou não pode fornecer resultados funcionais e estéticos suficientes para o tratamento. E a reabilitação dos incisivos laterais é necessária após a manutenção ortodôntica do espaço edêntulo. As alternativas de 
reabilitação com próteses variam, proporcionando maior satisfação psicológica, e devem ser consideradas. ${ }^{14}$ Dessa forma, muitos pacientes necessitam de diferentes tipos de próteses dentárias para reabilitar a área da fenda edêntula, normalmente a área dos incisivos laterais. ${ }^{15-16} \mathrm{~A}$ intervenção reabilitadora com próteses fixas se tornou uma necessidade quando a extensão edêntula permanece após a conclusão do tratamento ortodôntico e geralmente é considerada um elo final para a reabilitação oral completa em paciente com fissura labiopalatina. ${ }^{17} \mathrm{~A}$ possibilidade de tratamento escolhida para restaurar o local edêntulo em paciente com fissura labiopalatina é prescrita pela patência e extensão do defeito, presença de fenda labial e ou palatina não reparada, relação maxilomandibular e estado dos dentes remanescentes. ${ }^{18}$

Independente da prótese indicada a simetria dos arcos dentários deve ser avaliada, pois o que se visa com os tratamentos propostos é a oclusão adequada capaz de restabelecer estética, função, anatomia e inclusão social do paciente. 19-21

Estudos sobre a avaliação das dimensões dos arcos dentários de indivíduos com fissura labiopalatina após a ortodontia e sobre a estabilidade alcançada após o tratamento protético são carentes. Portanto, este estudo teve como objetivo comparar e correlacionar as dimensões lineares dos arcos dentários de pacientes com fissura labiopalatina após tratamento ortodôntico e protético com prótese parcial fixa com as dimensões de pacientes sem fissura labiopalatina imediatamente após a ortodontia. A hipótese a ser testada é que não há diferença de simetria entre os pacientes com e sem fissura labiopalatina após o tratamento ortodôntico e protético reabilitador.

\section{MATERIAL E MÉTODOS}

O estudo foi submetido e aprovado pelo Comitê de Ética em Pesquisa do $X X X X X X X X X X$ sob número $X X X X X X$. Os pacientes foram recrutados no próprio hospital e na XXXXXX. O cálculo amostral para detectar uma correlação simples entre o lado direito e esquerdo no arco maxilar e mandibular significante $(r=0,7)$, nível de significância de $5 \%(\alpha=0,05)$, com poder de $80 \%(\beta=0,8)$ foi de pelo menos 14 indivíduos por grupo.

\section{Características da amostra}


A simetria do arco dentário foi avaliada após tratamento reabilitador em 35 pacientes com idades entre 18 e 30 anos. Os indivíduos foram divididos em dois grupos distintos: $\mathrm{O}$ grupo dos pacientes com fissura labiopalatina reabilitados com uma prótese parcial fixa (GPPF) foi composto por 15 pacientes que receberam tratamento interdisciplinar no XXXXX e o grupo dos pacientes sem fissuras orofaciais (GNF), foi composto por 20 pacientes submetidos a tratamento ortodôntico.

Os critérios de inclusão dos pacientes selecionados para o estudo foram: Idade entre 18 e 30 anos, estarem regularmente em atendimento no XXXXX e XXXXX. Os participantes submetidos a cirurgia ortognática ou extração de pré-molares e portadores de próteses totais ou removíveis foram excluídos da pesquisa.

Todos os pacientes receberam tratamento ortodôntico semelhante, expansão rápida da maxila (ERM) com expansores Haas fixados aos caninos e segundos molares decíduos e uma barra lingual estendida aos primeiros molares permanentes. O protocolo de ativação foi o mesmo para todos os pacientes: um full swing por dia (2/4 pela manhã e 2/4 à noite) durante 7 dias. ${ }^{22}$ Após a expansão ativa, o dispositivo foi mantido como retenção por 6 meses, e todos os pacientes apresentaram as mesmas características oclusais iniciais, mordida cruzada anterior e/ou posterior.

\section{Medidas Lineares}

A análise foi feita utilizando modelos de gesso digitalizados através de um scanner de bancada a laser (R700TM; 3Shape A/S, Holmens Kanal 7, 1060, Copenhagen/Dinamarca) e toda amostra foi avaliada com o software Vectra Analysis Module (VAM) (VECTRA H1; Canfield Scientific, 4 Wood Hollow Road, Parsippany, Nova Jersey 07054/EUA). As medidas lineares foram baseadas em um estudo prévio $^{23}$. Foram mensuradas três medidas lineares: distância incisal-canina (INC) correspondente à distância linear do ponto incisal à ponta da cúspide canina (a); distância canino-molar (CM) correspondente à distância linear da ponta da cúspide canina à ponta da cúspide disto-vestibular do primeiro molar permanente (b); e a distância incisal-molar (INM) correspondente à distância linear da ponta incisal à ponta da cúspide disto-vestibular do primeiro molar permanente (c) (Fig 1). 

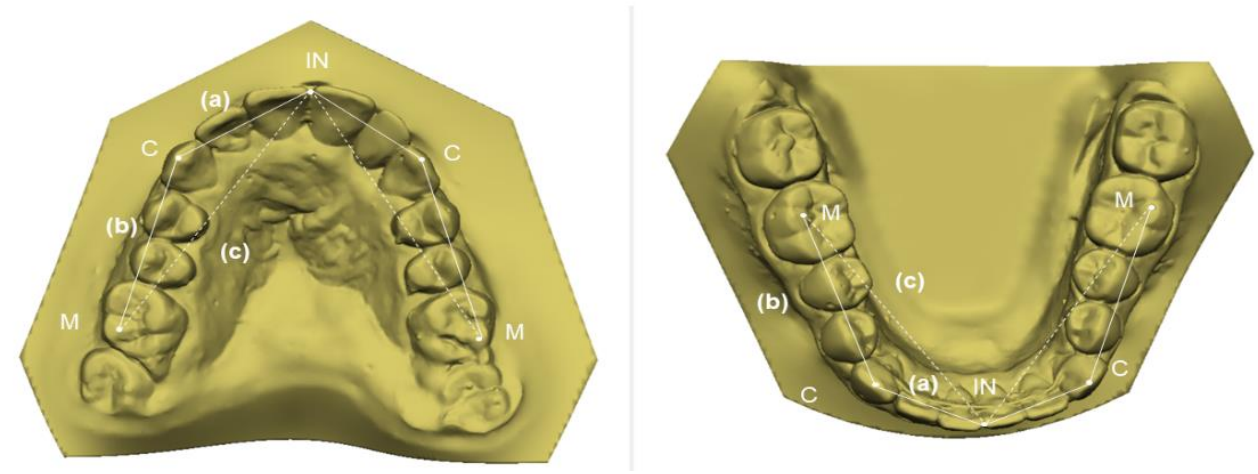

FIGURA 1. Medidas Lineares.

\section{Forma de análise dos resultados}

\section{Erro do método}

As medidas foram avaliadas duas vezes pelo mesmo examinador em $50 \%$ dos modelos digitalizados para analisar o erro do método, 15 dias após a primeira medida. Os erros sistemáticos foram avaliados através do teste t pareado e os erros aleatórios foram calculados. ${ }^{24}$ Não foram encontrados erros aleatórios e sistemáticos entre os dois tempos $(p \geq 0,05)$, sendo assim o examinador foi considerado calibrado.

\section{Análise Estatística}

A análise estatística foi realizada com o software Minitab (versão 19). Estatísticas descritivas foram realizadas para calcular a média, desvio padrão, mínimo, máximo, faixa e intervalo de confiança. O teste t-pareado e o coeficiente de correlação de Pearson ( $r$-value), significância $\alpha=0.05$, foram utilizados para comparação entre os lados direito e esquerdo do arco, de acordo com as variáveis grupo e sexo. O coeficiente de correlação (valor $r$ ) varia de $-1,0 \mathrm{a}+1,0$. Um r positivo indica que, à medida que uma variável aumenta, a outra também aumenta estabelecendo relação significativa quando $r \geq 0,6$ e quando o valor de $r$ for próximo de 0 isso indica que não há relação de associação entre as variáveis.

\section{RESULTADOS}

Verifica-se através do teste-t pareado $(P)$, que comparando os lados direito e esquerdo dos arcos dentários da maxila e mandíbula de pacientes com e sem fissura labiopalatina (GPPF e GNF), não há diferença estatisticamente significativa em 
nenhum deles $(P>0.05)$. E apesar de todos terem uma correlação positiva (análise de Correlação de Pearson - $r$ value, seguido de $p$ ), se mostrou quase nula, nas mensurações entre incisivos-caninos, tanto na maxila $(r=0,11)$ quanto na mandíbula $(r=0,26)$ de pacientes com fissura labiopalatina. O que não encontramos, bem como previsto, nos pacientes não fissurados. Todas as medidas nestes, com diferença estatisticamente não significativa e com uma correlaçao entre eles forte em todas as medidas, tanto na maxila (INC/r=0,63;CM/r=0,96;INM/r=0,89) quanto na mandíbula $(\mathrm{INC} / \mathrm{r}=0,76 ; \mathrm{CM} / \mathrm{r}=0,60 ; \mathrm{INM} / \mathrm{r}=0,67)($ Tabela 1$)$.

Tabela 1. Comparação e correlação das medidas lineares entre os lados direito e esquerdo, em pacientes com fissura labiopalatina (GPPF) e sem fissura (GNF)

\begin{tabular}{ccccc}
\hline Dimensões & Lado Direito & Lado Esquerdo & & \\
\hline GPPF (15) & Média/Dp $(\mathrm{mm})$ & Média/Dp $(\mathrm{mm})$ & T-pareado $(P)$ & $\begin{array}{c}\text { Correlação } \\
(\mathrm{r} \text {-value) }\end{array}$ \\
\hline Maxila & & & & \\
INC & $18.58 \pm 2.93$ & $18.39 \pm 2.59$ & 0.84 & 0.11 \\
CM & $23.68 \pm 3.45$ & $25.47 \pm 2.82$ & 0.05 & 0.47 \\
INM & $39.13 \pm 4.03$ & $40,07 \pm 3.36$ & 0.41 & 0.32 \\
Mandíbula & & & & \\
INC & $13.31 \pm 2.06$ & $13.92 \pm 1.34$ & 0.28 & 0.26 \\
CM & $24.32 \pm 3.27$ & $24.78 \pm 3.02$ & 0.55 & $\mathbf{0 . 5 6}$ \\
INM & $35.42 \pm 3.18$ & $33.11 \pm 3.43$ & 0.39 & $\mathbf{0 . 5 7}^{\star}$ \\
\hline GNF (20) & & & & \\
Maxila & & & & \\
INC & $18.41 \pm 1.20$ & $18.92 \pm 1.71$ & 0.11 & $\mathbf{0 . 6 3 ^ { \star * }}$ \\
CM & $27.21 \pm 1.78$ & $27.31 \pm 1.81$ & 0.36 & $\mathbf{0 . 9 6}$ \\
INM & $43.24 \pm 2.27$ & $42.98 \pm 2.64$ & 0.34 & $\mathbf{0 . 8 9}^{\star *}$ \\
Mandíbula & & & & \\
INC & $14.04 \pm 1.43$ & $14.23 \pm 0.87$ & 0.23 & $\mathbf{0 . 7 6}$ \\
CM & $25.35 \pm 3.31$ & $25.73 \pm 3.83$ & 0.59 & $\mathbf{0 . 6 0 ^ { \star * }}$ \\
INM & $36.96 \pm 3.28$ & $37.04 \pm 3.50$ & 0.89 & $\mathbf{0 . 6 7}^{\star}$ \\
\hline
\end{tabular}

INC: distância incisal-canino, CM: canino-molar e INM: incisal-molar/ *correlação moderada $(p<0.05) /$ Dp: desvio-padrão mm: milimetros

${ }^{\star *}$ correlação forte $(p<0.005)$.

Na comparação entre lados direito e esquerdo da maxila e mandíbula, nos sexos de pacientes com fissura labiopalatina (GPPF), observa-se que em nenhuma das mensurações existe diferença estatisticamente significativa perante ao teste $t$ pareado $(P>0.05)$ e há correlação positiva está presente em todas as mensurações (INC, CM E INM), tanto na maxila $(r=0,57 ; 0,59 ; 0,80)$ quanto na mandíbula $(r=0,82$; $r=0,69 ; r=0,78)$ porém apenas somente no sexo feminino. Ainda há de ressaltar que no sexo masculino, embora não haja diferença entre os lados segundo o teste $\mathrm{t}$ pareado, não houve correlação em nenhuma medida linear, não podendo assim concluir a tendencia a simetria do arco (Tabela 2). 
Tabela 2. Comparação e correlação das medidas lineares entre os lados direito e esquerdo em pacientes com fissura labiopalatina (GPPF) de acordo com o sexo

\begin{tabular}{ccccc}
\hline Dimensões & Lado Direito & Lado Esquerdo & & \\
\hline Sexo Feminino (7) & Média/Dp $(\mathrm{mm})$ & Média/Dp $(\mathrm{mm})$ & T-pareado $(P)$ & $\begin{array}{c}\text { Correlação } \\
(\mathrm{r} \text {-value })\end{array}$ \\
\hline Maxila & & & & \\
INC & $19.85 \pm 2.45$ & $18.95 \pm 1.45$ & 0.27 & $\mathbf{0 . 5 7 ^ { \star }}$ \\
CM & $23.78 \pm 3.87$ & $25.25 \pm 3.18$ & 0.27 & $\mathbf{0 . 5 9}^{\star}$ \\
INM & $40.18 \pm 4.56$ & $40.20 \pm 3.18$ & 0.98 & $\mathbf{0 . 8 0}^{\text {** }}$ \\
Mandíbula & & & & \\
INC & $12.88 \pm 2.45$ & $13.62 \pm 1.41$ & 0.24 & $\mathbf{0 . 8 2 ^ { \star * }}$ \\
CM & $25.34 \pm 2.84$ & $25.25 \pm 2.09$ & 0.91 & $\mathbf{0 . 6 9 ^ { \star * }}$ \\
INM & $35.65 \pm 3.83$ & $35.91 \pm 2.87$ & 0.77 & $\mathbf{0 . 7 8 ^ { \star * }}$ \\
\hline Sexo Masculino (8) & & & & \\
Maxila & & & & \\
INC & $17.47 \pm 2.99$ & $17.90 \pm 3.32$ & 0.80 & 0.1 \\
CM & $23.59 \pm 3.32$ & $25.67 \pm 2.68$ & 0.13 & 0.33 \\
INM & $38.21 \pm 3.55$ & $39.96 \pm 3.72$ & 0.39 & 0.1 \\
Mandíbula & & & & \\
INC & $13.69 \pm 1.72$ & $14.19 \pm 1.31$ & 0.61 & 0.53 \\
CM & $23.43 \pm 3.54$ & $24.37 \pm 3.77$ & 0.49 & 0.49 \\
INM & $35.24 \pm 2.75$ & $36.29 \pm 4.05$ & 0.44 & 0.47 \\
\hline
\end{tabular}

INC: distância incisal-canino, CM: canino-molar e INM: incisal-molar $/{ }^{*}$ correlação moderada ( $\left.\leq 0.05\right)$ / Dp: desvio-padrão mm: milimetros

${ }^{* *}$ correlação forte $(p \leq 0.005)$.

$\mathrm{Na}$ avaliação dos pacientes sem fissura labioapalatina (GNF) comprova-se a tendencia simetria quando comparados os lados direito e esquerdo da maxila e mandíbula dos sexos masculino e feminino, entretanto com uma correlação apesar de positiva, muito fraca na mandíbula dos homens $(\mathrm{CM} / \mathrm{r}=0,38$; INM/r=0,55) (Tabela 3$)$. 
Tabela 3. Comparação e correlação das medidas lineares entre os lados direito e esquerdo em pacientes sem fissura (GNF) de acordo com o sexo

\begin{tabular}{ccccc}
\hline Dimensões & Lado Direito & Lado Esquerdo & & \\
\hline $\begin{array}{c}\text { Sexo Feminino } \\
\text { (11) }\end{array}$ & Média/Dp $(\mathrm{mm})$ & Média/Dp $(\mathrm{mm})$ & T-pareado $(P)$ & $\begin{array}{c}\text { Correlação } \\
(\mathrm{r} \text {-value })\end{array}$ \\
\hline Maxila & & & & \\
INC & $19.34 \pm 0.97$ & $19.03 \pm 0.81$ & 0.08 & $\mathbf{0 . 8 3}^{\star \star}$ \\
CM & $26.57 \pm 1.51$ & $26.63 \pm 1.51$ & 0.53 & $\mathbf{0 . 9 7}^{\star \star}$ \\
INM & $42.68 \pm 1.91$ & $42.46 \pm 2.01$ & 0.10 & $\mathbf{0 . 9}^{\star \star}$ \\
Mandíbula & & & & \\
INC & $13.85 \pm 0.79$ & $14.02 \pm 0.76$ & 0.37 & $\mathbf{0 . 6 9}^{\star \star}$ \\
CM & $24.71 \pm 2.53$ & $24.55 \pm 2.71$ & 0.33 & $\mathbf{0 . 9 8}^{\star \star}$ \\
INM & $36.16 \pm 2.03$ & $35.72 \pm 1.99$ & 0.07 & $\mathbf{0 . 9 3}^{\star \star}$ \\
\hline Sexo Masculino (9) & & & & \\
\hline Maxila & & & & \\
INC & $19.49 \pm 1.50$ & $18.78 \pm 2.46$ & 0.30 & $\mathbf{0 . 6 2}^{\star \star}$ \\
CM & $27.99 \pm 1.84$ & $28.13 \pm 1.89$ & 0.51 & $\mathbf{0 . 9 4}$ \\
INM & $43.94 \pm 2.59$ & $43.63 \pm 3.28$ & 0.61 & $\mathbf{0 . 8 3 ^ { \star * }}$ \\
Mandíbula & & & & \\
INC & $14.28 \pm 1.29$ & $14.48 \pm 0.98$ & 0.46 & $\mathbf{0 . 8 0 ^ { \star * }}$ \\
CM & $26.13 \pm 4.09$ & $27.18 \pm 4.62$ & 0.53 & 0.38 \\
INM & $37.94 \pm 4.30$ & $38.65 \pm 4.35$ & 0.61 & 0.55 \\
\hline
\end{tabular}

INC: distância incisal-canino, CM: canino-molar e INM: incisal-molar/ *diferença estatística $(\mathrm{p} \leq 0.05)$ / Dp: desvio-padrão mm: milimetros

${ }^{* *}$ correlação forte $(p \leq 0.005)$.

\section{DISCUSSÃO}

O presente trabalho avaliou a simetria dos arcos dentários de pacientes com e sem fissura labiopalatina após tratamento ortodôntico/reabilitador utilizando uma prótese parcial fixa de 3 elementos na região da fenda. A metodologia utilizada foi a análise tridimensional em modelos digitalizados, baseada em estudos relevantes na literatura. ${ }^{8-13} \mathrm{~A}$ avaliação tridimensional através da estereofotogrametria é uma tecnologia confiável e validada. ${ }^{8}$

Pacientes com fissura labiopalatina necessitam de tratamento interdisciplinar desde o nascimento, iniciando pelas cirurgias primárias ${ }^{6}$, as quais restringem o crescimento maxilar ${ }^{25}$ e se tornam um desafio para a equipe interdisciplinar. Assim, o tratamento protético deve promover harmonia entre estética e função e incluir o paciente socialmente além de devolver qualidade de vida. Por esse motivo, é importante avaliar a simetria do arco após o tratamento reabilitador. Idealmente, um paciente com FLP deve ser reabilitado com tratamento ortodôntico e o espaço do incisivo lateral superior ausente deve ser fechado, movendo os dentes posteriores no lado da fissura; na maioria das vezes, o canino é movido para mesial. ${ }^{6}$ 
No entanto, pode levar a assimetria do arco dentário porque o dente ausente não é substituído. Outra solução é a substituição do dente perdido por uma prótese parcial fixa convencional. ${ }^{17}$ Por esse motivo, neste estudo, selecionamos participantes que receberam tratamento protético com prótese parcial fixa para substituição do dente ausente na região da fenda.

Nos dois grupos avaliados (GPPF e GNF) não se observou diferença, revelando uma tendência a simetria. Porém, no teste de correlação entre os lados, nos dois grupos, apenas o grupo GNF mostrou correlação significativa na maioria das medidas, sugerindo que esses pacientes tem tendência real ao arco simétrico. Esses resultados são corroborados pelos apresentados por outro autor, no qual a amostra incluiu pacientes não tratados ortodonticamente e em oclusão normal (classe I) ${ }^{23}$, indicando que os pacientes tratados no presente estudo após a ortodontia (GNF), atingiram um padrão simétrico mesmo partindo de uma má oclusão (Classe III). A hipótese testada foi rejeitada porque os pacientes com FLP, reabilitados com prótese parcial fixa, não apresentaram uma correlação forte principalmente na maxila, o arco associado a prótese.

No grupo GPPF, de acordo com o sexo, não se observou diferença estatística, mas as mulheres mostraram correlação positiva nos arcos dentários sendo os mais fortes INC mand, INMmax, INMmand com valores de r-value, respectivamente 0,82; 0,$80 ; 0,78$ (Tabela 2). Ao compararmos com o trabalho de Al-Zubair veremos que os

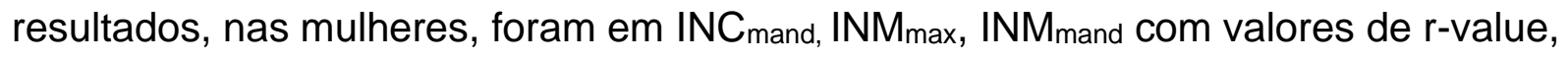
0,$91 ; 0,87 ; 0,87$, respectivamente, em pacientes sem fissura labiopalatina, com oclusão normal. ${ }^{23}$ Isso revela que mesmo em pacientes com condições adversas, como o grupo GPPF, é possível uma correlação forte no sexo feminino nas medidas acima dispostas. Trabalhos que avaliem a simetria de arcos de pacientes com fissura labiopalatina são escassos, dificultando a comparação entre resultados.

Em geral, é incomum observar um organismo com simetria perfeita nas duas metades do corpo $^{26}$, a assimetria verificada em questão é chamada de assimetria flutuante, a qual é caracterizada por diferenças entre os lados esquerdo e direito devido a erros aleatórios no desenvolvimento individual e pode ser vista como a variação do indivíduo em relação à assimetria direcional média. ${ }^{27}$

As assimetrias flutuantes do arco dentário foram reconhecidas como importantes fatores contribuintes para a etiologia da má-oclusão. ${ }^{28-30}$ Isso foi verificado no grupo GNF no trabalho em questão, visto que os pacientes inicialmente 
foram classificados com uma má-oclusão severa (classe III), porém ao final do tratamento ortodôntico, pudemos constatar que esse grupo de pacientes (GNF) apresentaram tendência a simetria tanto na maxila quanto na mandíbula, verificada pela não diferença estatística observada no teste-t pareado e correlação significante, em todas as medidas lineares (INC, CM, INM), resultados equivalentes a pacientes sem má-oclusão e não tratados ortodonticamente, avaliados por Al-Zubair. ${ }^{23}$

Quanto a avaliação no fator sexo, observamos resultados parcialmente semelhantes a estudo prévio, o qual verificou uma tendência a simetria no arco mandibular no sexo feminino ${ }^{31}$, embora o nosso estudo também tenha constatado 0 mesmo para a maxila. Os resultados para o sexo masculino do presente trabalho contrastam com os resultados obtidos por Ferrario el al., que independente de não ter constatado assimetria significante no sexo masculino, a região com maior tendência assimétrica foi a região anterior. ${ }^{31}$ No presente estudo, os resultados obtidos sugeriram essa assimetria na região posterior de mandíbula (CM, INM), pois nessas mensurações não verificamos uma correlação forte no sexo masculino ( $r=0,33 ; r=0,55$, respectivamente). A literatura relata que em média, os arcos se apresentam simétricos com índices médios variando de $0,28 \%$ a $4,57 \%$, revelando que certo grau de assimetria morfológica pode ser considerado um achado normal. ${ }^{32}$

Embora haja muitos trabalhos sobre assimetrias faciais, são escassas as pesquisas que avaliam a simetria dos arcos dentários. Em geral, não há consenso metodológico bem estabelecido nos estudos, pois alguns autores avaliam a simetria comparando a distância de alguns dentes de um lado, a linha média correspondente à rafe palatina mediana, com a distância dos dentes homólogos do outro lado à mesma linha que corresponde a rafe palatina mediana. ${ }^{33-38}$ Outros autores usam pontos dentais estratégicos e medidas lineares com base em triângulos semelhantes ${ }^{23,26}$, metodologia escolhida pelo presente trabalho pois a rafe palatina não se mostrou confiável como centro do palato como eixo de simetria ${ }^{23,39}$, ainda mais envolvendo pacientes com fissura labiopalatina que foram submetidos a palatoplastia.

Outros estudos são necessários para avaliação da simetria de arco após tratamento ortodôntico/reabilitador nos pacientes com fissura labiopalatina, pois o mesmo tem como objetivo minimizar a assimetria dento facial discrepante causada pela fissura. Uma das limitações do presente trabalho foi a seleção de uma amostra de conveniência, submetidos apenas ao tratamento de um centro específico 
(HRAC/USP), e para extrapolar os resultados obtidos no presente trabalho há a necessidade de realizar pesquisas semelhantes em outros centros.

\section{REFERÊNCIAS}

1 Shkoukani MA, Lawrence LA, Liebertz DJ, et al. Cleft palate: a clinical review. Birth Defects Res C Embryo Today 2014;102:333-42.

2 Will LA. Growth and development in patients with untreated clefts. Cleft Palate Craniofac J 2000;37:523-6.

3 World Health Organization. Global strategies to reduce the health-care burden of craniofacial anomalies. Geneva: World Health Organization, 2002. Disponível em: https://apps.who.int/iris/bitstream/handle/10665/42594/9241590386.pdf?sequence $=1$ \&isAllowed=y Acesso em: 27 jan. 2021 .

4 Freitas JA, Neves LT, Almeida AL, et al. Rehabilitative treatment of cleft lip and palate: experience of the Hospital for Rehabilitation of Craniofacial Anomalies/USP (HRAC/USP)--Part 1: overall aspects. J Appl Oral Sci 2012;20:9-15.

5 Martelli DR, Machado RA, Swerts MS, et al. Non syndromic cleft lip and palate: relationship between sex and clinical extension. Braz J Otorhinolaryngol 2012;78:116-20.

6 Freitas JA, Garib DG, Oliveira M, et al. Rehabilitative treatment of cleft lip and palate: experience of the Hospital for Rehabilitation of Craniofacial Anomalies-USP (HRAC-USP)--part 2: pediatric dentistry and orthodontics. J Appl Oral Sci 2012;20:268-81.

7 Wutzl A, Sinko K, Shengelia N, et al. Examination of dental casts in newborns with bilateral complete cleft lip and palate. Int J Oral Maxillofac Surg 2009;38:1025-9. 
8 Sforza C, Menezes M, Bresciani E, et al. Evaluation of a 3D stereophotogrammetric technique to measure the stone casts of patients with unilateral cleft lip and palate. Cleft Palate Craniofac J 2012;49:477-83.

9 Mello BZ, Fernandes VM, Carrara CF, et al. Evaluation of the intercanine distance in newborns with cleft lip and palate using 3D digital casts. J App/ Oral Sci 2013;21:437-42.

10 Huanca Ghislanzoni LT, Lineberger M, Cevidanes LH, et al. Evaluation of tip and torque on virtual study models: a validation study. Prog Orthod 2013;14:19.

11 Ugolini A, Cerruto C, Di Vece L, et al. Dental arch response to Haas-type rapid maxillary expansion anchored to deciduous vs permanent molars: a multicentric randomized controlled trial. Angle Orthod 2015;85:570-6.

12 Kuijpers MA, Chiu YT, Nada RM, et al. Three-dimensional imaging methods for quantitative analysis of facial soft tissues and skeletal morphology in patients with orofacial clefts: a systematic review. PLoS One 2014;9:e93442.

13 De Menezes M, Cerón-Zapata AM, López-Palacio AM, et al. Evaluation of a Three-Dimensional Stereophotogrammetric Method to Identify and Measure the Palatal Surface Area in Children With Unilateral Cleft Lip and Palate. Cleft Palate Craniofac J 2016;53:16-21

14 Malgaj T, Plut A, Eberlinc A. et al. Anterior esthetic rehabilitation of an alveolar cleft using novel minimally invasive prosthodontic techniques: a case report. Cleft Palate Craniofac J 2020:1055665620964709

15 Caballero JT, Pucciarelli MGR, Pazmiño VFC, Curvêllo VP, Menezes M, Sforza C, et al. 3D comparison of dental arch stability in patients with and without cleft lip and palate after orthodontic/rehabilitative treatment. J Appl Oral Sci 2019;27:e20180434. 
16 Pucciarelli MG, Toyoshima GHL, Oliveira TM, et al. Assessment of dental arch stability after orthodontic treatment and oral rehabilitation in complete unilateral cleft lip and palate and non-clefts patients using 3D stereophotogrammetry. BMC Oral Health 2020;20:(1):154.

17 Freitas JA, Almeida AL, Soares S, et al. Rehabilitative treatment of cleft lip and palate: experience of the Hospital for Rehabilitation of Craniofacial Anomalies/USP (HRAC/USP) - Part 4: oral rehabilitation. J Appl Oral Sci 2013;21:284-92.

18 Bhandari S. Clinical outcome of tooth-supported fixed partial dentures in unilateral cleft lip and palate patients: a case series. $J$ Indian Prosthodont Soc 2017;17(1):68-73.

19 Mølsted K, Dahl E. Asymmetry of the maxilla in children with complete unilateral cleft lip and palate. Cleft Palate J 1990;27:184-92.

20 Precious DS, Delaire J. Clinical observations of cleft lip and palate. Oral Surg Oral Med Oral Pathol 1993;75:141-51.

21 Park YH, Park S, Baek SH. Alignment strategy for constricted maxillary dental arch in patients with unilateral cleft lip and palate using fixed orthodontic appliance. J Craniofac Surg 2018;29:264-69.

22 Façanha AJ, Lara TS, Garib DG, et al. Transverse effect of Haas and Hyrax appliances on the upper dental arch in patients with unilateral complete cleft lip and palate: a comparative study. Dental Press J Orthod 2014;19:39-45.

23 Al-Zubair NM. Dental arch asymmetry. Eur J Dent 2014;8:224-8.

24 Dahlberg G. Statistical methods for medical and biological students. London: George Allen and Urwin; 1940.

25 Lilja J, Mars M, Elander A, et al. Analysis of dental arch relationships in Swedish unilateral cleft lip and palate subjects: 20-year longitudinal consecutive series 
treated with delayed hard palate closure. Cleft Palate Craniofac J 2006;43:60611.

26 Mutinelli S. Symmetry evaluation in the dental arch: the IXS index. Prog Orthod 2006;7:244-51.

27 Klingenberg CP. Analyzing fluctuating asymmetry with geometric morphometrics: concepts, methods, and applications. Symmetry 2015;7:843-934.

28 Garn SM, Lewis AB, Kerewsky RS. The meaning of bilateral asymmetry in the permanent dentition. Angle Orthod 1966;36:55-62.

29 Harris EF, Bodford K. Bilateral asymmetry in the tooth relationships of orthodontic patients. Angle Orthod 2007;77:779-86

30 Baydas B, Oktay H, Metin Dagsuyu I. The effect of heritability on Bolton tooth-size discrepancy. Eur J Orthod 2005;27:98-102.

31 Ferrario VF, Sforza C, Miani A Jr, et al. Dental arch asymmetry in young healthy human subjects evaluated by Euclidean distance matrix analysis. Arch Oral Biol 1993;38:189-94.

32 Ferrario VF, Sforza C, Colombo A, et al. Position and asymmetry of teeth in untreated dental arches. Int J Adult Orthodon Orthognath Surg 1993;8:277-85.

33 Lear CS. Symmetry analyses of the palate and maxillary dental arch. Angle Orthod 1968;38:56-62.

34 Maurice TJ, Kula K. Dental arch asymmetry in the mixed dentition. Angle Orthod 1998;68:37-44.

35 Kusnoto J, Evans CA, BeGole EA, et al. Orthodontic correction of transverse arch asymmetries. Am J Orthod Dentofacial Orthop 2002;121:38-45.

36 Cassidy KM, Harris EF, Tolley EA, et al. Genetic influence on dental arch form in orthodontic patients. Angle Orthod 1998;68:445-54. 
37 Langberg BJ, Arai K, Miner RM. Transverse skeletal and dental asymmetry in adults with unilateral lingual posterior crossbite. Am J Orthod Dentofacial Orthop $2005 ; 127: 6-16$.

38 Burstone CJ. Diagnosis and treatment planning of patients with asymmetries. Semin Orthod 1998;4(3):153-64.

39 Lundström A. Some asymmetries of the dental arches, jaws, and skull, and their etiological significance. Am J Orthod Dentofacial Orthop 1961;47:81-106. 


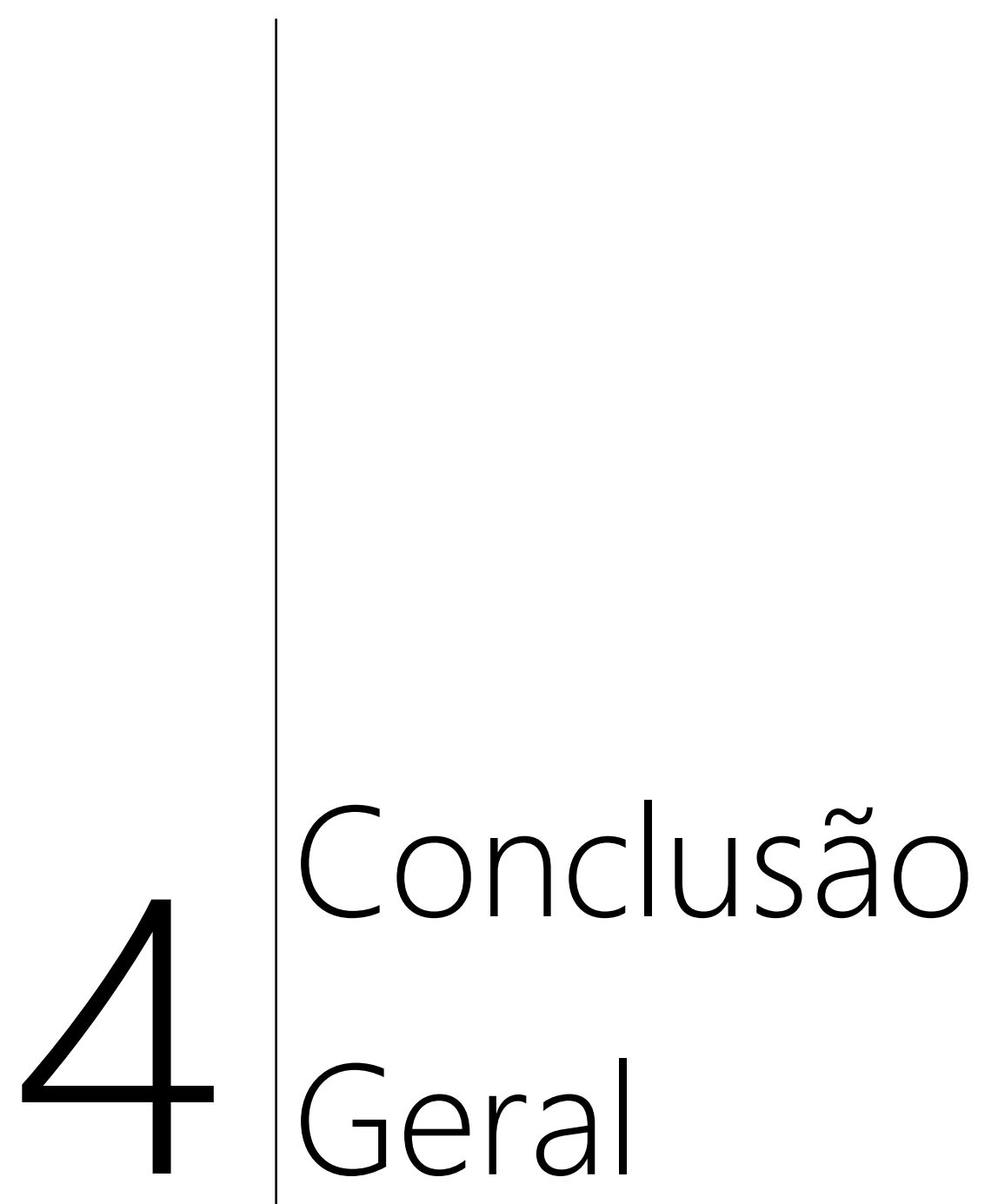





\section{CONCLUSÃO GERAL}

O presente estudo concluiu que os pacientes com fissura labiopalatina do grupo GPPF não apresentaram simetria na maxila. Isso ocorreu devido aos pacientes do sexo masculino $(n=8)$, os quais não apresentaram simetria em nenhum arco dentário, já nos indivíduos do sexo feminino desse grupo, todas as medidas foram consideradas simétricas em ambos os arcos. No grupo sem fissura labiopalatina (GNF) ambos os arcos foram considerados simétricos e apenas observou-se a não tendência a simetria na região posterior da mandíbula. 


\section{Referências}





\section{REFERÊNCIAS}

ABRAMSON, Z. R. et al. Radiology of cleft lip and palate: Imaging for the prenatal period and throughout life. Radiographics, v.35, n.7, p.2053-2063, Nov. 2015. doi: 10.1148/rg.2015150050.

ALONSO, N. et al. Fissuras labiopalatinas: protocolo de atendimento multidisciplinar e seguimento longitudinal em 91 pacientes consecutivos. Rev Bras Cirurg Plást, São Paulo, v.24, n.2, p.176-181, 2009.

AYNA, E.; BAŞARAN, E. G.; BEYDEMIR, K. Prosthodontic rehabilitation alternative of patients with cleft lip and palate (CLP): two cases report. Int J Dent, v.2009, p.515790, 2009. doi: 10.1155/2009/515790.

BARDACH, J. et al. Bone formation in the canine palate following partial resection.

Prog Clin Biol Res, New York, v.187, p.365-377, 1985.

BHANDARI, S. Clinical outcome of tooth-supported fixed partial dentures in unilateral cleft lip and palate patients: a case series. J Indian Prosthodont Soc., v.17, n.1, p.68-73, Jan./Mar. 2017. doi: 10.4103/0972-4052.197939.

BERTIER, C. E.; TRINDADE, I. E. K.; SILVA FILHO, O. G. Cirurgias primárias de lábio e palato. In: TRINDADE, I. E. K.; SILVA FILHO, O. G. (Coord.). Fissuras labiopalatinas: uma abordagem interdisciplinar. São Paulo: Editora Santos, 2007. p. 73-86.

BISHARA, S. E.; BURKEY, P. S.; KHAROUF, J. G. Dental and facial asymmetries: a review. Angle Orthod, Appleton, v.64, n.2, p.89-98, 1994. doi: 10.1043/00033219(1994)064<0089:DAFAAR>2.0.CO;2

BUTALI, A. et al. Genetic studies in the Nigerian population implicate an MSX1 mutation in complex oral facial clefting disorders. Cleft Palate Craniofac $\mathbf{J}$, Thousand Oaks, v.48, n.6, p.646-653, Nov. 2011. doi: 10.1597/10-133.

CABALLERO, J. T. et al. 3d comparison of dental arch stability in patients with and without cleft lip and palate after orthodontic/ rehabilitative treatment. J Appl Oral Sci, Bauru, v.27, p.e20180434, June 2019. doi: 10.1590/1678-7757-2018-0434.

CHEN, Z. et al. Asymmetric maxillary protraction for unilateral cleft lip and palate patients using finite element analysis. J Craniofac Surg, Burlington, v.26, n.2, p.388-392, Mar. 2015. doi: 10.1097/SCS.0000000000001337.

ESTEVES, A.; BOMMARITO, S. Avaliação da profundidade do palato e das dimensões do arco dentário superior em indivíduos com má oclusão e diferentes tipos faciais. Rev Dent Press Ortodon Ortop Facial, Maringá, v.12, n.4, p.84-98, 2007. Doi:10.1590/S1415-54192007000400010.

FREITAS, J. A. S. et al. Rehabilitative treatment of cleft lip and palate: experience of the Hospital for Rehabilitation of Craniofacial Anomalies/USP (HRAC/USP) - Part 1: 
overall aspects. J Appl Oral Sci, Bauru, v.20, n.1, p.9-15, Feb. 2012a.

FREITAS, J. A. S. et al. Rehabilitative treatment of cleft lip and palate: experience of the Hospital for Rehabilitation of Craniofacial Anomalies - USP (HRAC-USP) - Part 2: pediatric dentistry and orthodontics. J Appl Oral Sci, Bauru, v.20, n.2, p.268-281, Mar./Apr. 2012b.

FREITAS, J. A. S. et al. Rehabilitative treatment of cleft lip and palate: experience of the Hospital for Rehabilitation of Craniofacial Anomalies/USP (HRAC/USP) - Part 3: oral and maxillofacial surgery. J Appl Oral Sci, Bauru, v.20, n.6, p.673-679, Nov./Dec. 2012c.

FREITAS, J. A. S. et al. Rehabilitative treatment of cleft lip and palate: experience of the Hospital for Rehabilitation of Craniofacial Anomalies/USP (HRAC/USP) - Part 4: oral rehabilitation. J Appl Oral Sci, Bauru, v.21, n.3, p.284-292, 2013. doi: 10.1590/1679-775720130127.

FÜRHAUSER, R. et al. Evaluation of soft tissue around single-tooth implant crowns: the pink esthetic score. Clin Oral Implants Res, Copenhagen, v.16, n.6, p.639-644, Dec. 2005. doi:10.1111/j.1600-0501.2005.01193.x.

GNOINSKI, W. M.; RUTZ, G. A longitudinal cephalometric study from age 5 to 18 years on individuals with complete bilateral cleft lip and palate. J Craniofac Surg, Burlington, v.20, p.1672-1682, Sept. 2009. Suppl 2.

HUANCA GHISLANZONI, L. T. et al. Evaluation of tip and torque on virtual study models: a validation study. Prog Orthod, Copenhagen, v.14, n.19, p.14-19, July 2013. doi: 10.1186/2196-1042-14-19

JUODZBALYS, G.; WANG, H. L. Soft and hard tissue assessment of immediate implant placement: a case series. . Clin Oral Implants Res, Copenhagen, v.18, n.2, p.237-243, 2007. doi: 10.1111/j.1600-0501.2006.01312.x.

KATCHBURIAN, E.; ARANA, V. Histologia e embriologia oral. Rio de Janeiro: Guanabara Koogan, 2012.

KAZOR, C. E. et al. Implant plastic surgery: a review and rationale. J Oral Implantol, Abington, v.30, n.4, p.240-254, 2004. doi:10.1563/0.637.1.

KIM, S. W. et al. Change in upper lip height and nostril sill after alveolar bone grafting in unilateral cleft lip alveolus patients. J Plast Reconstr Aesthet Surg, v.65, n.5, p.558-536, May 2012. doi:10.1016/j.bjps.2011.11.046

KREY, K. F.; BÖRNGEN, J.; DANNHAUER, K. H. Three-dimensional analysis of the deciduous dentition of patients with bilateral cleft lip and palate and delayed cleft closure. J Orofac Orthop, v.70, n.3, p.237-246, May 2009. doi: 10.1007/s00056009-8814-8.

KUIJPERS, M. A. R. et al. Three-dimensional imaging methods for quantitative analysis of facial soft tissues and skeletal morphology in patients with orofacial clefts: 
a systematic review. PLoS ONE, San Francisco, v.9, n.4, p.e93442, Apr. 2014. doi: 10.1371/journal.pone.0093442.

LEAR, C. S. Symmetry analyses of the palate and maxillary dental arch. Angle Orthod, Appleton, v.38, n.1, p.56-62, Jan. 1968. doi: 10.1043/00033219(1968)038<0056:SAOTPA>2.0.CO;2.

LEWIS, C. W.; JACOB, L. S.; LEHMANN, C. U. The primary care pediatrician and the care of children with cleft lip and/or cleft palate. Pediatrics, Elk Grove Village II, v.139, n.5, p.e20170628, May 2017. doi: 10.1542/peds.2017-0628.

MALGAJ, T. et al. Anterior esthetic rehabilitation of an alveolar cleft using novel minimally invasive prosthodontic techniques: a case report. Cleft Palate Craniofac J, Thousand Oaks, p.1055665620964709, Oct. 2020. [in print].

MARTELLI, D. R. B. et al. Non sindromic cleft lip and palate: Relationship between sex and clinical extension. Braz J Otorhinolaryngol, São Paulo, v.78, n.5, p.116120, Oct. 2012.

MAURICE, T. J.; KULA, K. Dental arch asymmetry in the mixed dentition. Angle Orthod, Appleton, v.68, n.1, p.37-44, Feb. 1998. doi: 10.1043/00033219(1998)068<0037:DAAITM>2.3.CO;2.

MELLO, B. Z. F. et al. Evaluation of the intercanine distance in newborns with cleft lip and palate using 3D digital casts. J Appl Oral Sci, Bauru, v.21, n.5, p.437-442, Sept./Oct. 2013. doi: 10.1590/1679-775720130091

MENEZES, M. et al. Evaluation of a three-dimensional stereophotogrammetric method to identify and measure the palatal surface area in children with unilateral cleft lip and palate. Cleft Palate Craniofac J, Thousand Oaks, v.53, n.1, p.16-21, Jan. 2016. doi: 10.1597/14-076.

MOLSTED, K.; DAHL, E. Asymmetry of the maxilla in children with complete unilateral cleft lip and palate. Cleft Palate Craniofac J, Thousand Oaks, v.27, n.2, p.184-192, Apr. 1990. doi: 10.1597/1545-1569(1990)027<0184:aotmic>2.3.co;2.

OTERO, L. et al. A comparative study of facial asymmetry in Philippine, Colombian, and Ethiopian families with nonsyndromic cleft lip palate. Plast Surg Int, New York, v.2012, p.580769, Sept./Oct. 2012. doi: 10.1155/2012/580769.

PARK, Y. H.; PARK, S.; BAEK, S. H. Alignment strategy for constricted maxillary dental arch in patients with unilateral cleft lip and palate using fixed orthodontic appliance. J Craniofac Surg, Burlington, v.29, n.2, p.264-269, Mar. 2018. doi: 10.1097/SCS.0000000000004091.

PINTO, J. H. N.; LOPES, J. F. S. Reabilitação oral em prótese dentária. In: TRINDADE, I. E. K.; SILVA FILHO, O. G. (Coord.). Fissuras labiopalatinas: uma abordagem interdisciplinar. São Paulo: Editora Santos, 2007. p.261-274. 
PRECIOUS, D. S.; DELAIRE, J. Clinical observations of cleft lip and palate. Oral Surg Oral Med Oral Pathol, v.75, n.2, p.141-151, Feb. 1993. doi: 10.1016/00304220(93)90084-h.

PUCCIARELLI, M. G. R. et al. Assessment of dental arch stability after orthodontic treatment and oral rehabilitation in complete unilateral cleft lip and palate and nonclefts patients using 3D stereophotogrammetry. BMC Oral Health, London, v.20, n.1, p.154, May 2020. doi: 10.1186/s12903-020-01143-1.

ROSATI, R. et al. The occlusal plane in the facial context: inter-operator repeatability of a new three-dimensional method. Int J Oral Sci, Bangalore, v.4, n.1, p.34-37, Mar. 2012. doi: 10.1038/ijos.2012.2.

ROUSSEAU, P. et al. Effect of lip closure on early maxillary growth in patients with cleft lip and palate. JAMA Facial Plast Surg, Chicago, v.15, n.5, p.369-373, Sept./Oct. 2013. doi: 10.1001/jamafacial.2013.335.

SABARINATH, V. P. et al. Changes in maxillary alveolar morphology with nasoalveolar molding. J Clin Pediatr Dent, Birmingham, v.35, n.2, p.207--2012, 2010. doi: 10.17796/jcpd.35.2.f80u21362566qr34.

SCANAVINI, P. E. et al. Evaluation of the dental arch asymmetry in natural normal occlusion and class II malocclusion individuals. Dental Press J Orthod., Maringá, v.17, n.1, p.125-137, Feb. 2012. doi:10.1590/S2176-94512012000100016

SCHAEFER, K. et al. Dental arch asymmetry in an isolated Adriatic community. Am J Phys Anthropol, Hoboken, v.129, n.1, p.132-142, Jan. 2006. doi:

10.1002/ajpa.20224.

SHAH, S. M.; JOSHI, M. R. An assessment of asymmetry in the normal craniofacial complex. Angle Orthod, Appleton, v.48, n.2, p.141-148, Apr. 1978. doi: 10.1043/0003-3219(1978)048<0141:AAOAIT>2.0.CO;2

SEMB, G.; SHAW, W. C. Simonart's band and facial growth in unilateral clefts of the lip and palate. Cleft Palate Craniofac J, Thousand Oaks, v.28, n.1, p.40-48, Jan. 1991. doi: 10.1597/1545-1569_1991_028_0040_ssbafg_2.3.co_2.

SEMB, G. et al. The Eurocleft study: Intercenter study of treatment outcome in patients with complete cleft lip and palate. Part 1: Introduction and treatment experience. Cleft Palate Craniofac J, Thousand Oaks, v.42, n.1, p.64-68, Jan. 2005. doi: 10.1597/02-119.1.1.

SFORZA, C. et al. Evaluation of a 3D stereophotogrammetric technique to measure the stone casts of patients with unilateral cleft lip and palate. Cleft Palate Craniofac J, Thousand Oaks, v.49, n.4, p.477-483, July 2012. doi: 10.1597/10-207.

SHKOUKANI, M. A. et al. Cleft palate: a clinical review. Birth Defects Res. C Embryo Today, Hoboken, v.102, n.4, p.333-342, 2014. doi:10.1002/bdrc.21083.

SILVA FILHO, O. G.; FREITAS, J. A. S. Caracterização morfológica e origem 
embriológica. In: TRINDADE, I. E. K.; SILVA FILHO, O. G. (Coord.). Fissuras labiopalatinas: uma abordagem interdisciplinar. São Paulo: Editora Santos, 2007. p.17-50.

SILVA FILHO, O. G.; RAMOS, A. L.; ABDO, R. C. The influence of unilateral cleft lip and palate on maxillary dental arch morphology. Angle Orthod, Appleton, v.62, n.4, p.283-290, 1992. doi: 10.1043/0003-3219(1992)062<0283:TIOUCL>2.0.CO;2

ŠLAJ, M. et al. Longitudinal dental arch changes in the mixed dentition. Angle Orthod, Appleton, v.73, n.5, p.509-514, Oct. 2003. doi: 10.1043/00033219(2003)073<0509:LDACIT>2.0.CO;2.

SPINA, V. et al. Classificação das fissuras lábio-palatinas: sugestão de modificação. Rev Hosp Clin Faculd Med Univers Sao Paulo, São Paulo, v.27, n.1, p.5-6, 1972.

TOLAROVÁ, M. M.; CERVENKA, J. Classification and birth prevalence of orofacial clefts. Am J Med Genet, New York, v.75, n.2, p.126-137, Jan. 1998.

UGOLINI, A. et al. Dental arch response to Haas-type rapid maxillary expansion anchored to deciduous vs permanent molars: A multicentric randomized controlled trial. Angle Orthod, Appleton, v.85, n.4, p.570-576, July 2015. doi: 10.2319/041114269.1 .

WILL, L. A. Growth and development in patients with untreated clefts. Cleft Palate Craniofac J, Thousand Oaks, v.37, n.6, p.523-526, 2000. doi: 10.1597/15451569_2000_037_0523_gadipw_2.0.co_2

WORLD HEALTH ORGANIZATION. Global strategies to reduce the health-care burden of craniofacial anomalies. Geneva: World Health Organization, 2002. Disponível em: https://apps.who.int/iris/bitstream/handle/10665/42594/9241590386.pdf?sequence=1 \&isAllowed=y Acesso em: 27 jan. 2021.

WUTZL, A. et al. Examination of dental casts in newborns with bilateral complete cleft lip and palate. Int J Oral Maxillofac Surg, v.38, n.10, p.1025-1029, Oct. 2009. doi:10.1016/j.ijom.2009.04.023

YANG, C. J. et al. Impact of rapid maxillary expansion in unilateral cleft lip and palate patients after secondary alveolar bone grafting: review and case report. Oral Surg Oral Med Oral Pathol Oral Radiol, New York, v.114, n.1, p.e25-e30, July 2012. doi: 10.1016/j.tripleo.2011.08.030. 
Anexo 



\section{ANEXO A - Parecer Consubstanciado do Comitê de Ética em Pesquisa em Seres Humanos (CEP)}

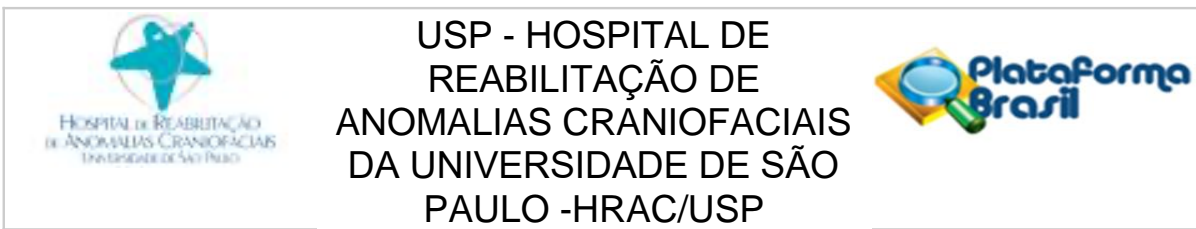

\section{PARECER CONSUBSTANCIADO DO CEP}

\section{DADOS DO PROJETO DE PESQUISA}

Título da Pesquisa: AVALIAÇÃO DA SIMETRIA DO ARCO DENTÁRIO DE PACIENTES COM E SEM FISSURA LABIOPALATINA APÓS TRATAMENTO ORTODÔNTICO/REABILITADOR PROTÉTICO.

Pesquisador: Simone Soares

Área Temática:

Versão: 2

CAAE: 36314820.6 .0000 .5441

Instituição Proponente: Hospital de Reabilitação de Anomalias Craniofaciais da USP

Patrocinador Principal: Financiamento Próprio

\section{DADOS DO PARECER}

Número do Parecer: 4.263 .528

\section{Apresentação do Projeto:}

Trata-se da segunda versão do projeto da pesquisa intitulada "AVALIAÇÃO DA SIMETRIA DO ARCO DENTÁRIO DE PACIENTES COM E SEM FISSURA LABIOPALATINA APÓS TRATAMENTO ORTODÔNTICO/REABILITADOR PROTÉTICO", apresentado ao CEP para análise dos aspectos éticos. Tem como pesquisadora responsável Simone Soares e fazendo parte da equipe de pesquisa José Antonio de Siqueira Laurenti e Andreia Fernandes Emilio. Este estudo retrospectivo transversal e observacional pretende analisar e correlacionar a simetria do arco dentário entre participantes com fissura labiopalatina e tratamento reabilitador completado e participantes sem fissura.A amostra será composta por trinta e cinco pacientes do Hospital de Reabilitação de Anomalias Craniofaciais (HRAC-USP)com idades entre 18 e 30 anos, que receberam tratamento interdisciplinar da fissura e após a ortodontia foram reabilitados com uma prótese parcial fixa ou coroa apoiada sobre implante, na área da fissura e 20 pacientes sem fissura labiopalatina e tratatados ortodonticamente. A análise da simetria dos arcos será realizada sobre modelos dentários previamente digitalizados, utilizando um scanner a laser modelo (R700TM; 3Shape) em todos os grupos e utilizando o programa Vectra Analysis Module (VAM) (VECTRA H1; Canfield Scientific). Serão avaliadas três medidas lineares: a distância incisal-canino, canino-molar e incisal-molar.

Endereço: Rua Silvio Marchione, 3-20 Bairro: Vila Nova Cidade Universitária

UF: SP Município: BAURU

CEP: $17.012-900$

Telefone: (14)3235-8421 Fax: (14)3234-7818 E-mail: cephrac@usp.br 
continuação

USP - HOSPITAL DE
REABILITAÇÃO DE
ANOMALIAS CRANIOFACIAIS
DA UNIVERSIDADE DE SÃO
PAULO -HRAC/USP

Continuaçāo do Parecer: 4.263.528

Recomendações:

Nenhuma

Conclusões ou Pendências e Lista de Inadequações:

Projeto ficou com pendência em relatoria anterior pela inadequação:

1 - Os Riscos $\theta$ Benefícios descritos na Plataforma Brasil $\theta$ no Projeto detalhado estavam diferentes $\theta$ necessitavam ser compatibilizados.Agora essa inconsistência foi sanada $\theta$ feita atualização do cronograma na Plataforma Brasil $\theta$ no Projeto detalhado.

Sugiro portanto ao CEP parecer aprovado.

Considerações Finais a critério do CEP:

Projeto Aprovado Ad Referendum que será referendado na Reunião de 29/09/2020

O pesquisador deve atentar que o projeto de pesquisa aprovado por este CEP refere-se ao protocolo submetido para avaliação. Portanto, conforme a Resolução CNS 466/12, o pesquisador é responsável por "desenvolver o projeto conforme delineado", se caso houver alterações nesse projeto, өste CEP deverá ser comunicado em emenda via Plataforma Brasil, para nova avaliação.

Cabe ao pesquisador notificar via Plataforma Brasil o relatório final para avaliação. Os Termos de Consentimento Livre $\theta$ Esclarecidos $\Theta / o u$ outros Termos obrigatórios assinados pelos participantes da pesquisa deverão ser entregues ao CEP. Os relatórios semestrais devem ser notificados quando solicitados no parecer.

Este parecer foi elaborado baseado nos documentos abaixo relacionados:

\begin{tabular}{|c|c|c|c|c|}
\hline Tipo Documento & Arquivo & Postagem & Autor & Situação \\
\hline $\begin{array}{l}\text { Informações Básicas } \\
\text { do Projeto }\end{array}$ & $\begin{array}{l}\text { PB_INFORMAÇŐEES_BÁSICAS_DO_P } \\
\text { ROJETO_1603048.pdf }\end{array}$ & $\begin{array}{c}01 / 09 / 2020 \\
11: 22: 18\end{array}$ & & Aceito \\
\hline Outros & $\begin{array}{l}\text { Projeto_Pesquisa_Simetria_cronograma } \\
\text { alterado.pdf }\end{array}$ & $\begin{array}{c}01 / 09 / 2020 \\
11: 21: 54\end{array}$ & Simone Soares & Aceito \\
\hline Outros & oficioderesposta.pdf & $\begin{array}{c}01 / 09 / 2020 \\
11: 19: 39\end{array}$ & Simone Soares & Aceito \\
\hline Folha de Rosto & fdr.pdf & $\begin{array}{c}05 / 08 / 2020 \\
10: 22: 49 \\
\end{array}$ & Simone Soares & Aceito \\
\hline Outros & justificativadispensatcle.pdf & $\begin{array}{c}01 / 08 / 2020 \\
18: 12: 30 \\
\end{array}$ & Simone Soares & Aceito \\
\hline Declaração de & Term_Aquiesc.pdf & $01 / 08 / 2020$ & Simone Soares & Aceito \\
\hline
\end{tabular}

Endereço: Rua Silvio Marchione, 3-20

Bairro: Vila Nova Cidade Universitária

UF: SP Município: BAURU

Telefone: (14)3235-8421 Fax: (14)3234-7818 $\quad$ E-mail: cephrac@usp.br 
continuação

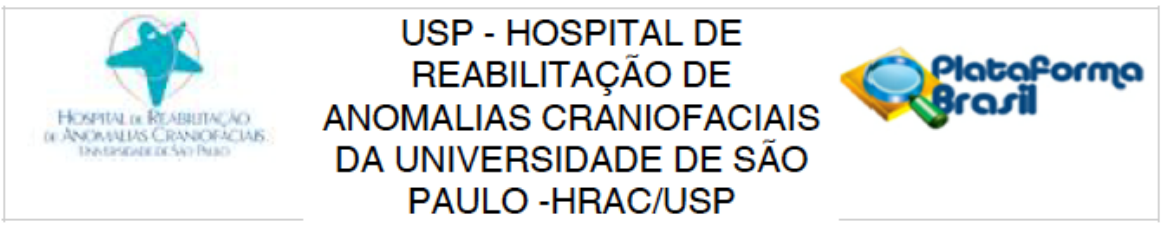

Continuaçāo do Parecer: 4.263.528

\begin{tabular}{|c|c|c|c|c|}
\hline concordância & Term_Aquiesc.pdf & 18:11:28 & Simone Soares & Aceito \\
\hline Outros & Term_Comp_Pesq_Resp.pdf & $\begin{array}{c}01 / 08 / 2020 \\
18: 10: 32\end{array}$ & Simone Soares & Aceito \\
\hline Outros & $\begin{array}{l}\text { Term_Comp_Tornar_Publico_Dest_Mat. } \\
\text { pdf }\end{array}$ & $\begin{array}{c}\text { 01/08/2020 } \\
18: 09: 02\end{array}$ & Simone Soares & Aceito \\
\hline Outros & Term_Perm_Uso_Registro.pdf & $\begin{array}{c}01 / 08 / 2020 \\
18: 06: 03\end{array}$ & Simone Soares & Aceito \\
\hline $\begin{array}{l}\text { Projeto Detalhado / } \\
\text { Brochura } \\
\text { Investigador }\end{array}$ & Projeto_Pesquisa_Simetria.pdf & $\begin{array}{c}01 / 08 / 2020 \\
18: 03: 59\end{array}$ & Simone Soares & Aceito \\
\hline Outros & Term_Comp_Conf_Aut_Dados.pdf & $\begin{array}{c}01 / 08 / 2020 \\
18: 03: 25\end{array}$ & Simone Soares & Aceito \\
\hline Outros & Form_Cadastro_HRAC.pdf & $\begin{array}{c}01 / 08 / 2020 \\
17: 58: 53\end{array}$ & Simone Soares & Aceito \\
\hline Outros & encaminhamento_ao_CEP.pdf & $\begin{array}{l}01 / 08 / 2020 \\
17: 57: 13\end{array}$ & Simone Soares & Aceito \\
\hline Orçamento & orcamento.pdf & $\begin{array}{l}28 / 07 / 2020 \\
14: 31: 40\end{array}$ & Simone Soares & Aceito \\
\hline
\end{tabular}

Situação do Parecer:

Aprovado

Necessita Apreciação da CONEP:

Não

BAURU, 08 de Setembro de 2020

Assinado por:

Renata Paciello Yamashita

(Coordenador(a))

Endereço: Rua Silvio Marchione, 3-20

Bairro: Vila Nova Cidade Universitária

UF: SP Municipio: BAURU

CEP: $17.012-900$

Telefone: (14)3235-8421

Fax: (14)3234-7818

E-mail: cephrac@usp.br 


\section{DECLARAÇÃO DE USO EXCLUSIVO DE ARTIGO EM DISSERTAÇÃO}

Declaramos estarmos cientes de que o trabalho Avaliação da simetria dos arcos dentários em pacientes com fissura labiopalatina reabilitados com prótese parcial fixa na região da fenda, após tratamento ortodôntico será apresentado na Dissertação da aluna Andreia Fernandes Emilio Laposta e que não foi e nem será utilizado em outra dissertação dos Programas de Pós-Graduação da FOB-USP.

Bauru, 20 de fevereiro de 2021.

Andreia Fernandes Emilio Laposta Nome do autor

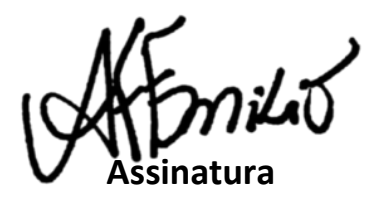

Simone Soares

Nome do autor

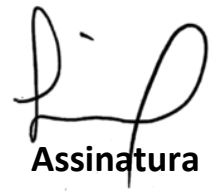




\section{DECLARATION OF EXCLUSIVE USE OF THE ARTICLE IN DISSERTATION}

We hereby declare that we are aware of the article Dental arches symmetry evaluation in cleft patients rehabilitated with a fixed partial denture in the cleft region, after orthodontic treatment will be included in Dissertation of the student Andreia Fernandes Emilio Laposta was not used and may not be used in other works of Graduate Programs at the Bauru School of Dentistry, University of São Paulo.

Bauru, 20 de fevereiro de 2021.

Andreia Fernandes Emilio Laposta Author

José Antônio de Siqueira Laurenti Author

Maria Giulia Rezende Pucciarelli Author

Jefferson Freire Cardoso

Author

Karin Hermana Neppelenbroek

Author

Simone Soares

Author

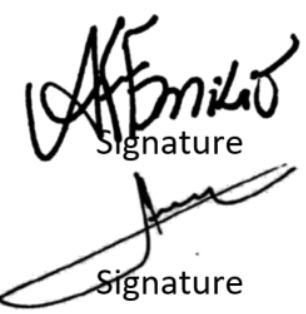

maria Givlia R. Puccionele

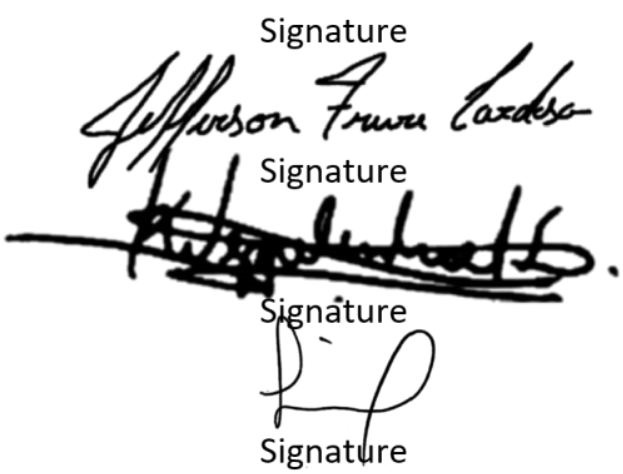

